\title{
Drying by Cavitation and Poroelastic Relaxations in Porous Media with Macroscopic Pores Connected by Nanoscale Throats
}

\author{
Olivier Vincent, ${ }^{1, *}$ David A. Sessoms, ${ }^{1}$ Erik J. Huber, ${ }^{2}$ Jules Guioth, ${ }^{1}$ and Abraham D. Stroock ${ }^{1,3, \dagger}$ \\ ${ }^{1}$ School of Chemical and Biomolecular Engineering, Cornell University, Ithaca, New York 14853, USA \\ ${ }^{2}$ Sibley School of Mechanical and Aerospace Engineering, Cornell University, Ithaca, New York 14853, USA \\ ${ }^{3}$ Kavli Institute at Cornell for Nanoscale Science, Cornell University, Ithaca, New York 14853, USA \\ (Received 25 February 2014; revised manuscript received 22 May 2014; published 25 September 2014)
}

\begin{abstract}
We investigate the drying dynamics of porous media with two pore diameters separated by several orders of magnitude. Nanometer-sized pores at the edge of our samples prevent air entry, while drying proceeds by heterogeneous nucleation of vapor bubbles_cavitation — in the liquid in micrometer-sized voids within the sample. We show that the dynamics of cavitation and drying are set by the interplay of the deterministic poroelastic mass transport in the porous medium and the stochastic nucleation process. Spatiotemporal patterns emerge in this unusual reaction-diffusion system, with temporal oscillations in the drying rate and variable roughness of the drying front.
\end{abstract}

DOI: 10.1103/PhysRevLett.113.134501

PACS numbers: 47.56.+r, 05.70.Ln, 47.55.dp, 89.75.-k

The desorption, or drying, of liquids from porous media is important in a variety of contexts, both in nature (e.g., the movement of water in plants [1]) and in technology (e.g., the synthesis [2] and characterization [3] of advanced materials). Studies of desorption have focused on the dynamics and pattern formation associated with drying [4-6] and the thermodynamics that define the shapes of desorption isotherms $[7,8]$. Despite this attention, uncertainties remain regarding the physical processes that govern desorption. It has been proposed that, rather than by receding of the liquid phase from the edges of the material, drying from porous media could occur by cavitation, i.e., the spontaneous formation of vapor bubbles either when the liquid tensile strength is attained [9] or by thermally activated nucleation in the metastable pore liquid [Fig. 1(a)] [10]. This process has been observed in simulations $[8,11]$ and has been proposed on several occasions to explain the shape of desorption isotherms in nanoscale porous media $[8,12,13]$ or the apparent emergence of drying events far from the evaporation front $[14,15]$. Yet, we are unaware of direct optical observation of desorption by cavitation or of an investigation of its effect on drying dynamics.

In this Letter, we present a tailored porous medium formed of microfabricated voids coupled to each other and the outside via a nanoporous substrate [Figs. 1(b) and 1(c)]. This extreme ink-bottle structure-large pore bodies connected via narrow throats [16] — has allowed us to observe the nucleation and growth of cavitation bubbles during drying [Fig. 1(d)] and to show that this process gives rise to interesting coupled drying dynamics that is tunable with geometry.

Drying occurs when a saturated porous medium is placed in a subsaturated atmosphere (relative humidity $p_{v} / p_{\text {sat }}<1$, where $p_{v}$ and $p_{\text {sat }}$ are the vapor pressure and its saturation value, respectively). At high relative humidity, evaporation results in the formation of menisci in the pores at the surface of the material, until local mechanical and chemical equilibrium are established, which occurs when the liquid pressure $P$ satisfies [17]
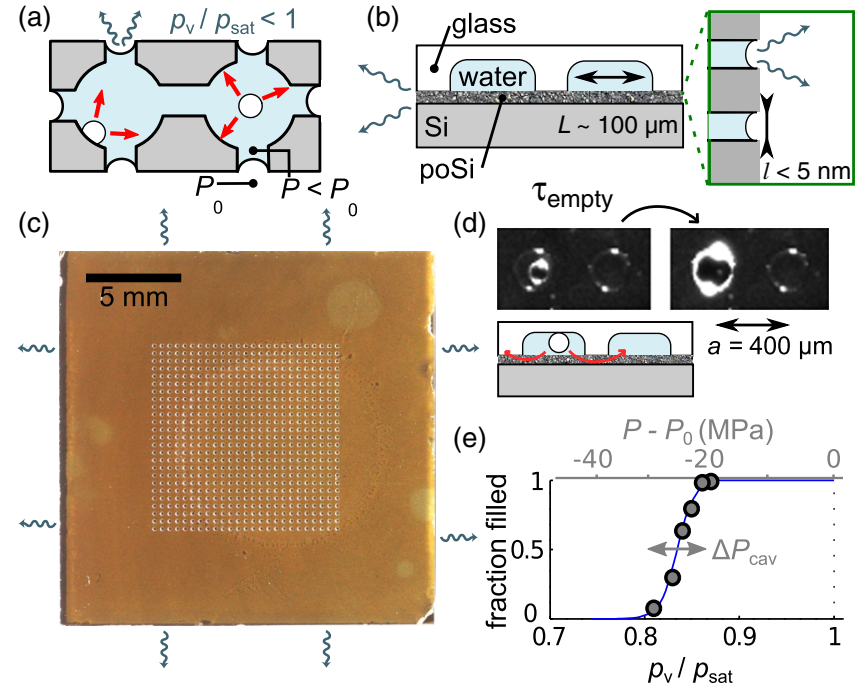

FIG. 1 (color online). (a) A porous medium with ink-bottle morphology may dry from inside by cavitation. (b) Schematic side view of our extreme ink-bottle geometry with microfabricated voids interconnected by porous silicon (poSi). (c) Photograph showing the top view of a sample with an array of $25 \times 25$ voids (bright dots) bonded to a layer of nanoporous silicon (orange background). Each void is dome shaped with a diameter of $200 \mu \mathrm{m}$ and a depth of $27 \mu \mathrm{m}$. (d) Optical micrographs of a cavitation event (top view) and a schematic drawing of the water fluxes (side view). The left micrograph shows the bubble immediately after cavitation; the right micrograph shows the empty void. (e) Survival curve (see the text). 


$$
P-P_{0}=\frac{R T}{v_{m}} \ln \left(\frac{p_{v}}{p_{\text {sat }}}\right)
$$

and

$$
P-P_{0}>-\frac{2 \sigma \cos \theta_{r}}{r_{p}},
$$

where $v_{m}=1.805 \times 10^{-5} \mathrm{~m}^{3} / \mathrm{mol}$ is the molar volume of the liquid, $R T$ is the thermal energy, $\sigma$ is the liquid surface tension, and $\theta_{r}$ is the receding contact angle of the meniscus in pores of radius $r_{p}$. At lower relative humidities, for which the pressure difference defined by (1) violates the inequality (2), the menisci will recede into the pores. This scenario is the classical process of drying by capillary invasion from the edges of the material [18].

Drying may also occur by a distinct mechanism: Equation (1) states that the pressure in the liquid phase is reduced relative to the standard pressure $P_{0}$, such that it is metastable with respect to the homogeneous or heterogeneous nucleation of bubbles of air and vapor $[9,19]$ [Fig. 1(a)]. This mode of drying by cavitation may be favored in "ink-bottle" geometries in which small pores prevent invasion of the menisci and permit the development of large stresses within the pore liquid ["pore blocking" via Eq. (2)], while larger pores inside allow the nucleation of bubbles [Fig. 1(a)].

Here, we report on an extreme version of the ink-bottle geometry in which nanometer- and micrometer-sized voids coexist; previous studies of bidisperse porous media have been limited to small ( $\leq$ twofold) differences in pore size $[7,12,20]$. Figures 1(b) and 1(c) present our system [21]: we formed discrete, micrometer-scale voids in the surface of glass via lithography; we formed a $5 \mu$ m-thick layer of interconnected, nanometer-scale pores (1-2 $\mathrm{nm}$ in radius) in the surface of silicon by anodization; and we bonded the glass and silicon anodically to couple the microvoids to each other and the outside environment via the nanopores.

We filled the evacuated samples by submerging them in liquid water at elevated pressure, then allowed them to dry in closed chambers with a controlled humidity, and followed the resulting dynamics by time-lapse photography. Equations (1) and (2) were always verified in our experiments, leaving cavitation as the only plausible mechanism for drying [21]. As seen in Fig. 1(d), we could follow discrete cavitation events within the microvoids during drying.

We first characterized the stability of the bulk liquid water within the microvoids by lowering the vapor pressure in step increments and counting the number of full voids after 1 day of equilibration [Fig. 1(e)]. Across samples, the shapes of the survival curves were similar, with probability $1 / 2$ of cavitation for $p_{v} / p_{\text {sat }}=0.83-0.86$, corresponding to liquid pressures in the range $P_{\text {cav }}=-20$ to $-24 \mathrm{MPa}$ from Eq. (1) (width $\Delta P_{\text {cav }}=2-5 \mathrm{MPa}$ ). This value is less negative than expected for homogeneous nucleation in water $\left(P_{\text {cav }} \sim-140 \mathrm{MPa}\right)$ [19], suggesting a heterogeneous process, but matches closely most measurements of $P_{\text {cav }}$ in water [27]. The exact nucleation mechanism is, however, not important for the dynamics of drying.

Figure 2(a) presents a typical drying sequence for $p_{v} / p_{\text {sat }}=0.84$ such that the boundary liquid pressure was $P_{b} \simeq-24 \mathrm{MPa}$ [Eq. (1)]. Drying proceeded in a mixed fashion, with both coherent, frontlike progression from the edges to the center and discrete events far away from the front. This observation suggests that the progression of cavitation was controlled by an interplay of deterministic mass transport and stochastic cavitation events that allow drying deep within the sample.

More surprisingly, despite the steady nature of the applied driving force, we found that cavitation and emptying of the voids proceeded in a punctuated manner, with bursts of one to seven events separated by periods with no events [Fig. 2(b)]. This progression of bursts persisted until all voids were empty [21] and showed long time correlations [Fig. 2(c)]. This emergent behavior suggests links to the dynamics in other out-of-equilibrium systems [28] such as the periodic relaxations in a continuously driven sand pile [29], which motivates the search for mechanisms of positive and negative feedback.

We considered but could exclude positive feedback based on acoustic emissions from cavitation events [21]. The period between successive bursts $[\simeq 15 \mathrm{~min}$; see Figs. 2(b) and 2(c)] was similar to the time scale for bubble growth after cavitation $\left[\tau_{\text {empty }} \simeq 3.5 \mathrm{~min}[21]\right.$; see Fig. 1(d)], which suggests that the bursting behavior arose from a hydraulic coupling due to the release of water into the surrounding medium during emptying. (a)

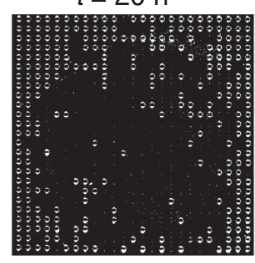

(b)

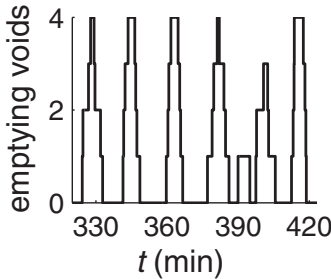

$t=40 \mathrm{~h}$
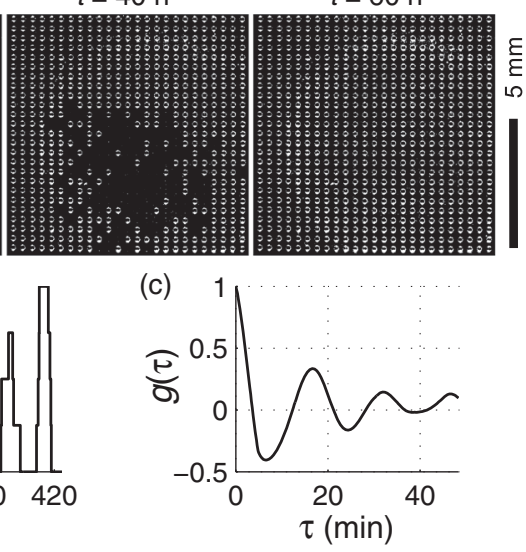

FIG. 2. Drying dynamics. (a) Optical micrographs showing state of voids in a sample as in Fig. 1(c) (only the array of voids was imaged) during drying at $p_{v} / p_{\text {sat }}=0.84$. Empty voids appear bright. Evaporation occurred from all four edges of the samples. (b) Number of voids in the process of emptying as a function of time. (c) Autocorrelation of the time series as in (b) for all 625 cavitation events. 
In order to check this hypothesis, we developed a model coupling deterministic mass transport and stochastic cavitation kinetics. We treat fluid transport within the porous silicon as poroelastic diffusion [30]:

$$
\frac{\partial P}{\partial t}=C \nabla^{2} P
$$

where $C=\kappa K_{\text {liq }} / \phi=5 \times 10^{-8} \mathrm{~m}^{2} / \mathrm{s}$ is the poroelastic diffusivity, $\kappa=1.44 \times 10^{-17} \mathrm{~m}^{2} /($ Pa.s $)$ is the Darcy permeability of porous silicon, $\phi=0.6$ is its porosity, and $K_{\text {liq }}=2.2 \mathrm{GPa}$ is the bulk modulus of liquid water.

We describe cavitation kinetics in each void by the classical nucleation theory $[19,27]$, leading to the following expression for the rate of nucleation:

$$
k_{\mathrm{cav}}(t)=\Gamma_{0} V \exp \left(-\frac{E_{\text {nucl }}(P)}{k_{B} T}\right)
$$

where $\Gamma_{0}$ is a kinetic prefactor, $V$ is the void volume, and $k_{B}$ is Boltzmann's constant. In the energy barrier for nucleation, $E_{\text {nucl }}=16 \pi \sigma^{3} /\left[3\left(P-p_{\text {sat }}\right)^{2}\right], \sigma \simeq 0.02 \mathrm{~N} / \mathrm{m}$ is an effective surface tension with a value typical of those used to match the observed kinetics of cavitation of water in a variety of experiments [17,27,31]. In order to account for spatial heterogeneities, we also allowed $\sigma$ to vary with a narrow distribution $(\Delta \sigma / \sigma \sim 0.02-0.07)$. We adjusted $\sigma$ and $\Delta \sigma$ to fit the experimental survival curves [Fig. 1(e), blue line].

We numerically solved Eq. (3) with an explicit finite difference scheme [32] for the evolution of the pressure field, $P(x, y, t)$. The four edges of the sample were maintained at a fixed liquid pressure $P_{b}$ set by the outside humidity [Eq. (1)]. At each time step $\Delta t$, the probability of nucleation $k_{\mathrm{cav}} \times \Delta t$ was calculated in each void [Eq. (4)], and cavitation was triggered with a Monte Carlo scheme. If cavitation occurred, the pressure in the void was set to $P=$ $p_{\text {sat }}$ (liquid-vapor equilibrium) until the void was empty of liquid. After emptying, the vapor pressure in the void was allowed to evolve in local equilibrium [Eq. (1)] with the liquid pressure $P$ in the neighboring region.

The sequence in Figs. 3(a)-3(d) presents snapshots of the calculated pressure field during a burst of four cavitation events under conditions matching the experiment shown in Fig. 2 [33]. When a void cavitates, its pressure jumps from the negative value in the surrounding matrix to $p_{\text {sat }}$ [red pixels in Fig. 3(a)]. This transition generates outward flow, emptying of the void, and the growth of a resaturated zone in the surrounding matrix [Fig. 3(b), white lines]. Outside of this zone, strong metastability persists and further cavitation events can occur [Fig. 3(b), events 1 and 2]; inside this zone, metastability is reduced and the nucleation rate is negligible. The resaturated zones of multiple events grow and merge [Fig. 3(c)] until further cavitation is suppressed throughout the sample [Fig. 3(d)]. Once the cavitated voids have emptied, they cease to act as (a)

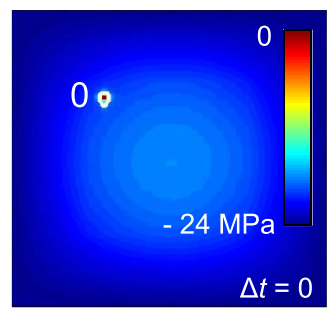

(c)

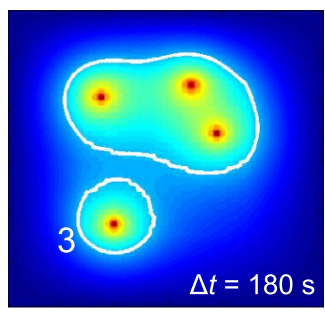

(e)

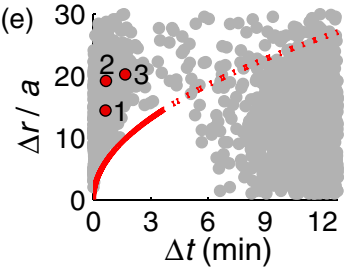

(b)

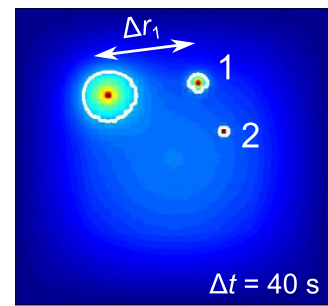

(d)

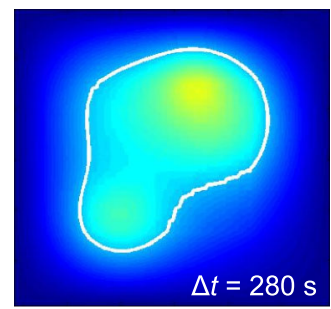

(f)

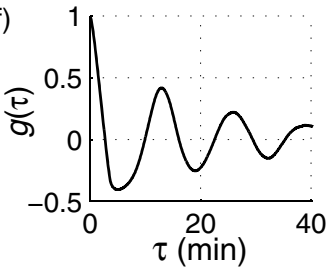

FIG. 3 (color online). Time evolution of the simulated pressure field in a $25 \times 25$ voids sample as in Fig. 2(a). (a) First cavitation event within a burst, labeled 0. (b) Growing exclusion zones (tracked by white contour lines at $-17 \mathrm{MPa}$ ). (c) Exclusion zones merge. (d) Retraction of the exclusion zones. (e) Spatiotemporal representation of the drying dynamics for the full simulation, showing spatial $(\Delta r)$ versus temporal $(\Delta t)$ separations for all pairs of cavitation events. Events 1-3 from (a)-(d) (with event 0 at origin) are represented as red dots. The red line (continuous for $\Delta t<\tau_{\text {empty }}$ and dashed afterwards) corresponds to $\Delta r=\alpha \sqrt{C \Delta t}$ with $\alpha=1.7$. (f) Autocorrelation function of all emptying events in frame (e).

sources for the rehydration of their surroundings; the pressure in the sample begins to decrease again due to continued evaporation at the boundary, and a new cycle of cavitation (that is, a burst) can start.

Figure 3(e) presents the spatiotemporal statistics of all cavitation events during the simulation. A pair of cavitation events separated by a time $\Delta t$ can exist only outside of an exclusion zone (red line), the spatial extent of which grows as $\Delta r_{\text {excl }} \sim \sqrt{C \Delta t}$ during a time scale on the order of the average emptying time $\tau_{\text {empt }}$. The growth in $t^{1 / 2}$ arises from poroelastic diffusion [Eq. (3)].

The similarity of the autocorrelation function (which measures the bursting behavior) of simulated events [Fig. 3(f)] to that observed experimentally [Fig. 2(c)] supports the conclusion that the time-dependent response emerges due to this suppressive effect based on hydraulic coupling. As will be shown below, this coupling can be tuned experimentally by varying the geometry of the inkbottle system.

The emergence of this complex response from the coupling of diffusion [Eq. (3)] and nonlinear reaction 
[Eq. (4)] points to a particular analogy with the relaxation of metastable states in reaction-diffusion dynamics that leads, for example, to pattern formation during the precipitation of supersaturated solutions [34].

We turn to the identification of grouped parameters that control spatiotemporal drying patterns in these ink-bottle materials, with pressure diffusion and cavitation kinetics as the basic ingredients. First, we define a nondimensional interaction between voids to capture the negative feedback illustrated in Fig. 3. If the spatial range of the poroelastic coupling $\left(\Delta r_{\text {excl }} \sim \sqrt{C \times \tau_{\text {empt }}}\right)$ is smaller than the distance $a$ between voids, the interaction should vanish. We thus define a nondimensional parameter comparing these two length scales:

$$
\zeta=\tau_{\mathrm{empt}} \times C / a^{2},
$$

which is also equal to the ratio of emptying time to the time $\tau_{\text {diff }}=a^{2} / C$ for pressure information to travel from one void to its neighbor.

Second, we define a nondimensional driving force for cavitation:

$$
\delta=\left(P_{\text {cav }}-P_{b}\right) / \Delta P_{\text {cav }},
$$

where $P_{b}$ is the liquid pressure at the boundary defined by the external humidity [Eq. (1)] and $P_{\text {cav }}$ and $\Delta P_{\text {cav }}$ parametrize the survival curve [Fig. 1(e)]. We expect $\delta$ to be a natural measure of the roughness of the drying front [21]: for strong driving forces $(\delta \gg 1)$, we expect sharp fronts, controlled by the diffusion of the boundary pressure into the sample; for weak driving forces (small positive and negative values of $\delta$ ), we expect spatially random events controlled by the kinetics of cavitation.

We investigated experimentally the effect of the two dimensionless parameters established above. First, the coupling parameter $\zeta$ [Eq. (5)] was varied by using samples with the same intervoid spacing, $a=400 \mu \mathrm{m}$, but with a void volume that was $\sim 200$-fold smaller than that of the samples discussed up to this point. This geometrical change lowered the interaction parameter from $\zeta=70$ (Fig. 4, top row) to $\zeta=0.1$ (Fig. 4, bottom row) by decreasing the emptying time by a factor of 700 . As predicted, the negative feedback between cavitation events was dramatically reduced, as can be seen in the nearly complete disappearance of the exclusion zones for both the strong and low driving force cases (Fig. 4, bottom). Furthermore, the time sequence of cavitation showed no measurable temporal correlations.

Second, to investigate the impact of driving force $\delta$, we exposed the same samples to different humidities (Fig. 4; $\delta$ increases from left to right). As predicted, we observed that the drying front became much sharper for higher values of $\delta$, with little effect on the hydraulic coupling (as seen in spatiotemporal maps). This evolution of the front shape is similar to that reported for evaporative drying of porous media $[4,35]$. The important distinction here is that drying

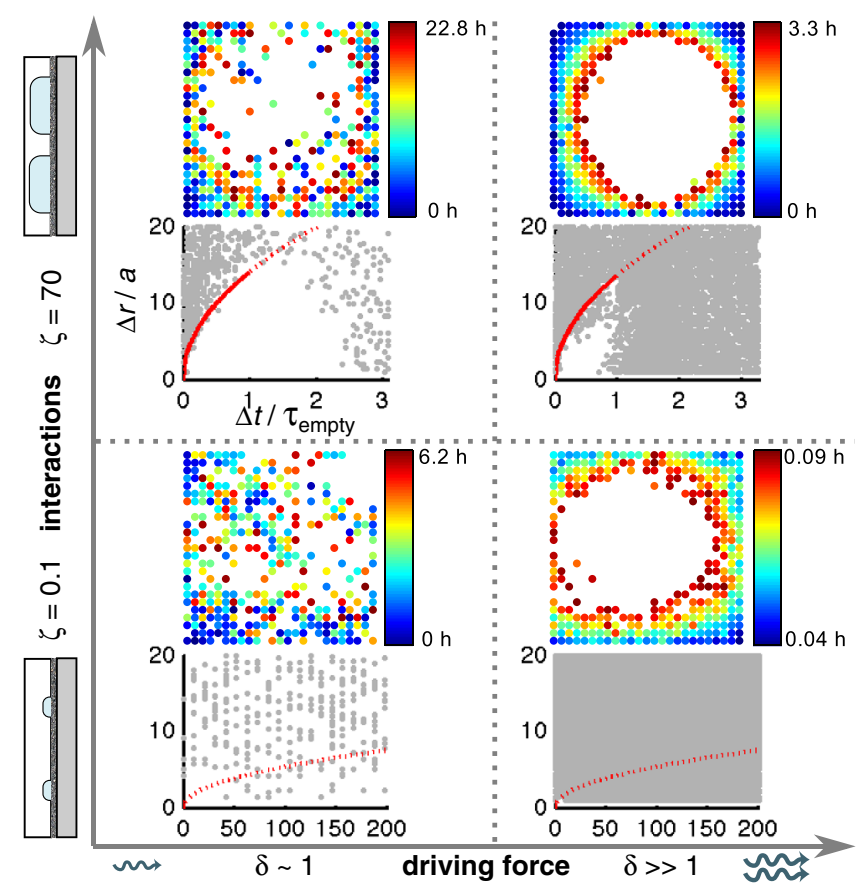

FIG. 4 (color online). Experimental state diagram of drying dynamics as a function of driving force and interactions between voids. The upper diagram in each quadrant is a spatial representation of the drying front when half of the voids had cavitated (color indicates event time). The lower diagram is the measure of temporal correlations introduced in Fig. 3(e) (the different data point densities reflect the impact of geometry or driving force on drying speed). The top left experiment is the one reported in Fig. 2.

occurs via cavitation at localized, spatially random points beyond the drying front, without percolation of a vapor path to the surface. Our experiments thus provide the first direct, experimental support of the proposal that this evolution of the pattern of drying with the driving force may result from cavitation $[14,15]$.

Finally, we note that our simulations based on Eqs. (3) and (4) reproduce the state diagram in Fig. 4 well [21], providing further support for the relevance of our model.

In conclusion, we have used extreme ink-bottle geometries to provide direct optical observation of drying of a porous medium by cavitation. We have further elucidated aspects of the emergent dynamics of this process and shown that we can explain them quantitatively with a model that couples poroelastic transport and cavitation kinetics. This mechanism represents a new route to a nonlinear reaction-diffusion process, with mechanical rather than molecular diffusion and with nonlinear kinetics coming from nucleation rather than from reaction. We expect that similar coupled dynamics should occur in highly heterogeneous porous media, such as the ones found in rocks or plant tissues. In the geological context, the propagation of drying stresses could lead to patterns of nucleation of gases from supersaturated solutions that are formed, for example, during the sequestration of carbon dioxide [36]. In plant 
tissues, the hydraulic interactions upon drying and cavitation might play a role in the physiological response to drought, as suggested by early results from Dixon, Grace, and Tyree [37]. We expect that microfabricated structures of the type presented here will provide useful avenues for the exploration of these and other phenomena.

The authors thank Eugene Choi for the measurement of nitrogen isotherms. This work was supported by the National Science Foundation (CBET-0747993 and CHE0924463), the Air Force Office of Scientific Research (FA9550-09-1-0188), and the Camille Dreyfus TeacherScholar Awards program and was performed in part at the Cornell NanoScale Facility, a member of the National Nanotechnology Infrastructure Network (National Science Foundation; Grant No. ECCS-0335765).

*orv3@cornell.edu †ads10@cornell.edu

[1] A. D. Stroock, V. V. Pagay, M. A. Zwieniecki, and N. M. Holbrook, Annu. Rev. Fluid Mech. 46, 615 (2014).

[2] C. J. Brinker and G. W. Scherer, Sol-Gel Science (Academic, New York, 1990).

[3] E. P. Barrett, L. G. Joyner, and P. P. Halenda, J. Am. Chem. Soc. 73, 373 (1951).

[4] T. M. Shaw, Phys. Rev. Lett. 59, 1671 (1987).

[5] M. Prat, Chem. Eng. J. (Lausanne) 86, 153 (2002).

[6] P. Lehmann, S. Assouline, and D. Or, Phys. Rev. E 77, 056309 (2008).

[7] D. Wallacher, N. Künzner, D. Kovalev, N. Korr, and K. Knorr, Phys. Rev. Lett. 92, 195704 (2004).

[8] P. I. Ravikovitch and A. V. Neimark, Langmuir 18, 9830 (2002).

[9] R. K. Schofield, Discuss. Faraday Soc. 3, 105 (1948).

[10] D. Or and M. Tuller, Water Resour. Res. 38, 19 (2002).

[11] L. Sarkisov and P. A. Monson, Langmuir 17, 7600 (2001).

[12] A. Grosman and C. Ortega, Langmuir 27, 2364 (2011).

[13] F. Bonnet, M. Melich, L. Puech, and P. E. Wolf, Europhys. Lett. 101, 16010 (2013).

[14] A. Sarkar, S. Chaudhuri, S. Wang, F. Kirkbir, and H. Murata, J. Sol-Gel Sci. Technol. 2, 865 (1994).

[15] G. W. Scherer and D. M. Smith, J. Non-Cryst. Solids 189, 197 (1995).
[16] L. H. Cohan, J. Am. Chem. Soc. 66, 98 (1944).

[17] T. D. Wheeler and A. D. Stroock, Nature (London) 455, 208 (2008)

[18] G. W. Scherer, J. Am. Ceram. Soc. 73, 3 (1990).

[19] P. G. Debenedetti, in Metastable Liquids: Concepts and Principles, edited by J. M. Prausnitz and L. Brewer (Princeton University Press, Princeton, NJ, 1996).

[20] L. Bruschi, G. Mistura, L. Liu, W. Lee, U. Gösele, and B. Coasne, Langmuir 26, 11894 (2010).

[21] See Supplemental Material at http://link.aps.org/ supplemental/10.1103/PhysRevLett.113.134501, which includes Refs. [22-26], for details on experiments, model, simulations, and expanded discussion.

[22] J. Chirife and S. L. Resnik, J. Food. Sci. 49, 1486 (1984).

[23] A. Rogacs, J. E. Steinbrenner, J. A. Rowlette, J. M. Weisse, X. L. Zheng, and K. E. Goodson, J. Colloid Interface Sci. 349, 354 (2010).

[24] F. Caupin and A. D. Stroock, The Stability Limit and Other Open Questions on Water at Negative Pressure, Liquid Polymorphism, edited by H. E. Stanley (John Wiley \& Sons, Inc., Hoboken, New Jersey, 2013) pp. 51-80.

[25] T. D. Wheeler and A. D. Stroock, Langmuir 25, 7609 (2009).

[26] J. Bear, Dynamics of Fluids in Porous Media (Courier Dover Publications, Mineola, New York, 1988).

[27] F. Caupin and E. Herbert, C.R. Phys. 7, 1000 (2006).

[28] M. C. Cross and P. C. Hohenberg, Rev. Mod. Phys. 65, 851 (1993).

[29] H. M. Jaeger, C.-h. Liu, and S. R. Nagel, Phys. Rev. Lett. 62, 40 (1989).

[30] M. A. Biot, J. Appl. Phys. 12, 155 (1941).

[31] K. Davitt, A. Arvengas, and F. Caupin, Europhys. Lett. 90, 16002 (2010).

[32] J. Crank, The Mathematics of Diffusion (Oxford University Press, New York, 1979).

[33] See Supplemental Material at http://link.aps.org/ supplemental/10.1103/PhysRevLett.113.134501 for the video corresponding to the simulation on Fig. 3.

[34] K. H. Stern, Chem. Rev. 54, 79 (1954).

[35] M. Prat and F. Bouleux, Phys. Rev. E 60, 5647 (1999).

[36] H. E. Huppert and J. A. Neufeld, Annu. Rev. Fluid Mech. 46, 255 (2014).

[37] M. A. Dixon, J. Grace, and M. T. Tyree, Plant Cell Environ. 7, 615 (1984). 